\title{
Cost-effectiveness of therapist-delivered online cognitive-behavioural therapy for depression: randomised controlled trial
}

\author{
Sandra Hollinghurst, Tim J. Peters, Surinder Kaur, Nicola Wiles, Glyn Lewis \\ and David Kessler
}

\section{Background}

Therapist-delivered online cognitive-behavioural therapy (CBT) has been found to be effective for depression in primary care.

\section{Aims}

To determine the cost-effectiveness of online CBT compared with usual care.

\section{Method}

Economic evaluation at 8 months alongside a randomised controlled trial. Cost to the National Health Service (NHS), personal costs, and the value of lost productivity, each compared with outcomes based on the Beck Depression Inventory and quality-adjusted life-years (QALYS). Incremental analysis indicated the NHS cost per QALY gain.

\section{Results}

Online CBT was more expensive than usual care, although the outcomes for the CBT group were better. Cost per QALY gain based on complete case data was $£ 17173$, and $£ 10083$ when missing data were imputed.

\section{Conclusions}

Online CBT delivered by a therapist in real time is likely to be cost-effective compared with usual care if society is willing to pay at least $f 20000$ per QALY; it could be a useful alternative to face-to-face CBT.

\section{Declaration of interest}

None.
There is a growing awareness of the healthcare burden of depression. ${ }^{1}$ The associated cost burden is less well documented: two important studies vary in their estimates, although there is agreement that this is substantial. In the clinical practice guidelines published by the British Psychological Society, ${ }^{2}$ the authors suggest a figure of about $£ 424$ per patient for direct treatment costs during a 6-month period. The more recent study by the King's Fund ${ }^{3}$ offers an estimate of annual service costs of $£ 2085$ per patient. Both studies agree that the cost of lost productivity due to depression is considerable; the Kings' Fund study puts this at $\mathfrak{E 7 . 5}$ billion a year. The scale of the combined healthcare and general economic burden has stimulated an interest in the effectiveness and cost-effectiveness of different types of care for patients with depression. Primary care treatment of depression has relied largely on antidepressant medication, although there are concerns about the levels of prescribing ${ }^{4}$ and, for some patients, safety ${ }^{5}$ of some antidepressants. Psychological therapies are popular and patient satisfaction is greater than with usual care; ${ }^{6}$ cognitive-behavioural therapy (CBT) is often preferred as it has the largest evidence base. ${ }^{7}$ However, the full potential of CBT is not exploited in primary care - partly because of a lack of therapists despite the current commitment to a training programme, ${ }^{8}$ but also because therapists are not always available at a time and place convenient to the patient. One proposed solution is a computerised CBT programme, which is effective and cost-effective compared with usual care, ${ }^{9,10}$ and less expensive than face-to-face CBT; however, this is inflexible and cannot be adapted to individual patient needs. Here, we assess the cost-effectiveness of a novel way of delivering CBT that combines the technological approach of CCBT with the personal advantages of CBT with a therapist. Therapists and patients use an instant messaging service to communicate online with each other, in real time, using typewritten responses.

\section{Method}

We carried out an economic evaluation alongside a randomised controlled trial (RCT) to establish the cost-effectiveness of therapist-delivered internet CBT compared with usual care for primary care patients with depression. Evidence for the clinical effectiveness of this intervention has been published elsewhere. ${ }^{11}$

\section{Sample}

We recruited patients aged between 18 and 75 who were identified in primary care as having a new episode of depression. This was defined as a score of 14 or more on the Beck Depression Inventory $(\mathrm{BDI})^{12}$ and an ICD-10 diagnosis of depression using the Revised Clinical Interview Schedule (CIS-R). ${ }^{13}$ Participants were recruited from 55 general practices in Bristol, London and Warwickshire between October 2005 and February 2008, and were randomised to online CBT in addition to usual care or to usual care while on an 8-month waiting list for online CBT. For full details of the trial methods see Kessler et al. ${ }^{11}$

\section{The intervention}

Participants receiving online CBT were offered up to ten sessions of 55 minutes, to be completed within 4 months from the date of randomisation if possible. Each participant was assigned their own therapist for the duration of the study. The psychologists worked for the organisation Psychologyonline (www.psychologyonline. co.uk); all were CBT-trained and had experience of providing therapy online. Participants and therapists typed free text into the computer, with messages sent instantaneously, using only this means of communication. The intervention offered was CBT as described by Judith Beck. ${ }^{14}$ Therapist adherence to this model 
was assessed using the Revised Cognitive Therapy Scale ${ }^{15}$ and is described elsewhere. ${ }^{11}$ Participants were reassessed after 8 months, and those on the waiting list who were still eligible by virtue of their BDI score were offered the intervention at that time.

\section{Outcome measures}

Clinical effectiveness was assessed using the BDI score in two ways: recovery was defined as a score of less than 10 , and we recorded the continuous score at follow-up as well as baseline. To assess cost-effectiveness we compared the extra cost of providing online CBT with the extra benefit. In addition to the BDI-related outcomes, benefit was also measured using quality-adjusted lifeyears (QALYs). The QALYs take account of both the quantity and quality of life generated by a healthcare intervention so are, in theory, a common currency that can be used to compare the cost-effectiveness of any intervention with that of others. In line with the recommendation of the National Institute for Health and Clinical Excellence (NICE), we used the EQ-5D ${ }^{16}$ to obtain the QALYs. ${ }^{17}$ This widely used measure of health-related quality of life is easily administered and comprises five dimensions of health: mobility, self-care, usual activities, pain and discomfort, and anxiety and depression. The EQ-5D was completed at baseline, 4 months and 8 months, and we used the tariff obtained from a UK general population survey to value the health states. ${ }^{18}$

\section{Resource use}

We measured resources used by participants over the 8-month period between randomisation and final follow-up: NHS resources, personal costs incurred by participants, and lost productivity due to time off work were each measured separately. Participants recorded their use of resources in a diary. They were asked to note details of all primary and community healthcare consultations and prescribed medication, and any secondary care received that was related to their mental health. Many primary and community care contacts are complex, involving more than one moderating factor and it is difficult to identify which are due to mental health issues - either solely or in part. For that reason we included all primary and community contacts. Secondary care is relatively expensive and fairly uncommon in this group of patients and the disadvantage of including all secondary care contacts is that by including non-relevant resources these may overshadow all other resource use, hence the focus on mental health-related secondary care contacts. We also asked about use of Social Services.

Personal costs included private sector healthcare, over-thecounter drugs, social and domestic help, travel costs and out-of-pocket loss of earnings (e.g. relating to self-employment or agency work). Participants also recorded the number of working days lost because of their depression whether or not they incurred a loss of earnings. Two separate identical diaries were completed for the period between baseline assessment and 4 months, and between 4 and 8 months. Monthly emails were used to remind participants of the importance of completing and returning the diaries; these were supplemented by telephone calls and home visits where necessary. The number of online CBT sessions used by each participant was recorded by the trial study team.

\section{Valuing the resource use}

All healthcare resources were valued using unit costs derived from nationally available data sources. These are shown in Table 1. Primary and community care was valued using Curtis ${ }^{19}$ and the costs of using walk-in centres and NHS Direct were based on national evaluations. ${ }^{20,21}$ For secondary care we used the National
Schedule of Reference Costs ${ }^{22}$ and medication was valued using the British National Formulary. ${ }^{23}$ The cost of personal expenditure was reported directly except for travel by car, which was costed using the AA schedule of motoring costs. ${ }^{24}$ Time off work was valued using median gross weekly earnings by age and gender, ${ }^{25}$ scaled down by $20 \%$ to allow for the fact that not all work time is productive. ${ }^{26}$ In the trial, the therapists were paid $\mathfrak{£} 40$ per hour and Psychologyonline was paid $£ 11$. However, the overhead charge of $\mathfrak{E} 11$ was negotiated specifically for the trial and this would normally have been $£ 20$, so this figure has been used in the analysis. In addition, we have allowed for a supervision cost making a total of $£ 62.50$ per hour (Table 1 ). All costs were valued in British pounds sterling $(\mathfrak{E})$ at 2007 prices, adjusted for inflation where necessary.

\section{Data analysis}

We investigated resources used by participants in each group using frequencies, means and medians for each category. Resource use was combined with unit costs to estimate the mean total cost per participant in each group, by category. Quality-adjusted lifeyears were computed using the area under the curve approach, which weights the 8 -month period by quality of life measured on a scale of 0 to 1 . For example, a QALY score of 0.667 would indicate perfect health throughout this period, whereas a score of 0.5 indicates an average quality of life of 0.75 .

A cost-consequences matrix was used to examine, for each group, mean cost per participant by category, the number of patients recovering, the mean BDI score and QALYs. Cost-effectiveness from the perspective of the NHS was assessed by comparing the difference between the cost of treating

\section{Table 1 Data sources and unit costs}

\begin{tabular}{|c|c|}
\hline & Unit cost, $f$ \\
\hline \multicolumn{2}{|l|}{ General practitioner $^{\mathrm{a}}$} \\
\hline Surgery & 30.00 \\
\hline Telephone consultation & 21.00 \\
\hline Home visit & 55.00 \\
\hline \multicolumn{2}{|l|}{ Practice nurse ${ }^{a}$} \\
\hline Surgery & 8.00 \\
\hline Telephone consultation & 5.60 \\
\hline Home visit & 12.00 \\
\hline Counsellor $^{a}$ & 34.00 \\
\hline Health visitor $^{\mathrm{a}}$ & 27.66 \\
\hline Occupational health ${ }^{a}$ & 14.00 \\
\hline Psychiatric doctor at surgery ${ }^{a}$ & 16.00 \\
\hline Phlebotomist $^{\mathrm{a}}$ & 8.00 \\
\hline Physiotherapist ${ }^{a}$ & 14.00 \\
\hline \multicolumn{2}{|l|}{ Walk-in centre ${ }^{b}$} \\
\hline Nurse & 30.18 \\
\hline Doctor & 48.18 \\
\hline NHS Direct ${ }^{\mathrm{b}}$ & 19.37 \\
\hline Accident and emergency ${ }^{c}$ & 61.78 \\
\hline Out-patient visits $^{c}$ & By clinic \\
\hline Prescribed medication $^{d}$ & By item \\
\hline Mileage $^{\mathrm{e}}$ & 0.44 \\
\hline Time off work ${ }^{f}$ & By age and gender \\
\hline $\begin{array}{l}\text { Intervention: online cognitive-behavioural } \\
\text { therapy per session }\end{array}$ & 62.50 \\
\hline $\begin{array}{l}\text { a. Unit costs of health and social care } 2007 .{ }^{19} \\
\text { b. Derived from national evaluations. }{ }^{19,20} \\
\text { c. Department of Health reference costs. }{ }^{21} \\
\text { d. According to the British National Formulary. } \\
\text { e. According to the AA schedule. } \\
\text { f. According to the Office of National Statistics. }{ }^{25}\end{array}$ & \\
\hline
\end{tabular}


participants in the online CBT group and those in the waiting list group with the difference in benefits. This is expressed as cost per extra patient recovering and cost per QALY gain.

We used sensitivity analyses to investigate the effects of three areas of uncertainty regarding the cost and QALY estimates. First, we assessed the effect of missing data. We imputed missing observations of cost and QALYs using the multiple imputation by chained equation procedure in Stata release $10{ }^{27}$ Twenty data-sets were generated and ten switching procedures were undertaken in the model: randomisation group; the cost of primary care, secondary care, and medication, each at 4 and 8 months; and QALYs at 4 and 8 months. The analysis was re-run using imputed data and the results compared with the base case estimates. Second, we addressed the fact that although participants were asked to record secondary care specifically related to their depression, several reported out-patient appointments that appeared not to be for mental health issues. In the sensitivity analysis, these were excluded to gauge the effect on the overall results. Third, we looked at the cost of the therapy sessions. In the base case analysis we used the rate paid to therapists taking part in the trial, plus an extra allowance for company overheads and supervision; in the sensitivity analysis, we estimated the maximum cost that could be paid for therapy for the cost per QALY to remain within the thresholds used by NICE (i.e.

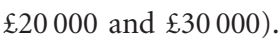

Patient variation in resource use and effectiveness can be captured by confidence intervals of the cost and outcome estimates separately; however, confidence intervals cannot be constructed for ratios so we used the technique of bootstrapping to capture this type of uncertainty for the estimate of cost per QALY. Using the original data, 5000 replicates of the costeffectiveness ratio were generated to indicate the scale of combined cost and effectiveness variability. These replicates were used to construct a cost-effectiveness acceptability curve, which indicates the probability that online CBT is cost-effective for any given level of willingness to pay in terms of cost per QALY. We also estimated the net monetary benefit gained from adopting the intervention for given values of cost per QALY that society might be willing to pay $(£ 20000 \text { and } £ 30000)^{28}$ and the probability that the net monetary benefit is positive for these values. There was no need to discount costs or outcomes as the time frame for the study was 12 months. All analyses were carried out using Microsoft Excel (Office 11) and Stata 10 on a personal computer (Windows XP).

\section{Results}

A total of 297 participants were recruited to the trial, 149 to the online CBT group and 148 to the usual care group. More women than men took part $(68 \% v .32 \%)$ and ages ranged from 18 to 74 with a mean (s.d.) age of 34.9 (11.6) years. Scores on the BDI were similar across the groups; $5 \%$ were classed with mild depression, $26 \%$ with moderate depression and $69 \%$ with severe depression. Mean (s.d.) BDI score was 33.2 (8.8). At the 8-month follow-up, we obtained BDI data for $210(70 \%)$ individuals and data to calculate QALYs for 165 (56\%) individuals; NHS cost data were complete for $137(46 \%)$ and we had information on time off work for $140(47 \%)$. Information on personal costs was poorly completed, with fewer than $20 \%$ of participants completing all sections of the diary at both time points.

\section{Resource use}

National Health Service resource use is shown in Table 2. These results are based on participants for whom we had any information for the whole period, so the number of observations varies across categories. Nearly all (93\%) patients who returned completed diaries used primary care services at least once during the 8 months, although for $76 \%$ of these it was less than once a month on average. The mean number of contacts was higher in the waiting list group than the CBT group, although variability in the estimates is too large to be able to draw reliable conclusions about a difference between them. The number of accident and emergency (A\&E) visits and out-patient appointments was small in both groups. Reasons for visiting $A \& E$ were varied: four were for stomach pain, five were alcohol- or drug-related. Out-patient services used most frequently were psychology, pain clinics and orthopaedics. Medication use was similar across the two groups; just over half of the participants reported a prescription for antidepressants during the 8 -month period. Of the 149 participants allocated to receive the online CBT, 19 (13\%) did not use the service at all and $47(32 \%)$ used the full allocation of ten sessions. Five sessions took place after the 8-month follow-up; we adopted a conservative approach and included the cost of these in the analysis.

Table 2 National Health Service resource use by allocation group

$\frac{\text { Waiting list }}{n} \frac{\text { Intervention }}{n} \frac{n}{\%}$

\section{Primary care}

appointments 0

$1-5$

6-10

$11+$

Total

Mean (s.d.)

Median (IQR)

A\&E visits

0
1

1
2
3

Total

Mean (s.d.)

Median (IQR)

Out-patient attendances

0

1

$2+$

Total

Mean (s.d.)

Median (IQR)

46

$31 \quad 48.4$

20

9

64

$$
31.3
$$

14.1

100.0

6.0 (4.3)

$5(3-8)$

$6 \quad 8.1$

$43 \quad 58.1$

$21 \quad 28.4$

$4 \quad 5.4$

74

100.0

4.9 (3.9) 4 (2-7)

Medication - all items

0

$1-5$

6-10

$11+$

Total

$57 \quad 89.1$

89.1
7.8

7.8
0.0

3.1

2

100.0

$0.17(0.58)$

$0(0)$

$67 \quad 89.3$

89.3

10.7

0.0

0.0

100.0

$0.11(0.31)$ $0(0)$

0

84.4

$\begin{array}{rr}54 & 84.4 \\ 3 & 4.7\end{array}$

10.9

100.0

64

0.41 (1.11) $0(0)$

0

0

7

\begin{tabular}{l} 
Total \\
Mean (s.d.) \\
Median (IQR) \\
\hline
\end{tabular}

Online CBT

0

1-5

6-10

$11+$

Total

Mean (s.d.)

Median (IQR)

$A \& E$, accident and emergency department; CBT, cognitive-behavioural therapy; IQR, interquartile range. 
Table 3 Time off work: number of working days off

\begin{tabular}{|lccccc|} 
& \multicolumn{2}{c}{ Waiting list } & & \multicolumn{2}{c|}{ Intervention } \\
\cline { 2 - 3 } \cline { 5 - 6 } & $n$ & $\%$ & & $n$ & $\%$ \\
\hline $1-5$ & 40 & 60.6 & & 54 & 73.0 \\
\hline $6-10$ & 5 & 7.6 & & 4 & 5.4 \\
\hline $11-15$ & 6 & 9.1 & & 1 & 1.4 \\
\hline $16-20$ & 2 & 3.0 & & 1 & 1.4 \\
\hline $21-25$ & 1 & 1.5 & & 3 & 4.1 \\
\hline $26-30$ & 1 & 1.5 & & 3 & 4.1 \\
\hline $31-35$ & 2 & 3.0 & & 0 & 0.0 \\
\hline $36-40$ & 0 & 3.0 & & 2 & 2.7 \\
\hline $41+$ & 7 & 10.6 & & 2 & 2.7 \\
\hline Total & 66 & 100.0 & 74 & 100.0 \\
\hline Mean (S.d.) & & $12.7(27.2)$ & & $7.6(17.9)$ \\
\hline Median (IQR) & & $0(0-9)$ & & $0(0-1)$ \\
\hline IQR, interquartile range. & & & & \\
\hline
\end{tabular}

A third of participants reported having some time off work because of their depression (Table 3). Of these, most were off work for less than 2 weeks, although 11 reported being off for more than 6 weeks. Overall, those in the waiting list group had more time off than those in the CBT group; fewer of them had no time off and their absences were likely to be longer. As only two participants identified extra use of Social Services, these have not been included in the analysis.

\section{Costs and consequences}

Table 4 shows the disaggregated costs and outcomes estimated using all available data by category and group; the number of observations varies across category, which means each must be viewed independently. The cost of healthcare services used per patient is lower in the CBT group than in the waiting list group,

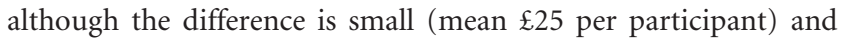
the confidence interval of $-\mathfrak{E} 87$ to $\mathfrak{E} 137$ suggests there is no evidence of a difference between the two groups. Personal expenditure on travel, over-the-counter preparations, private treatments and domestic help was higher in the intervention group, although there is considerable uncertainty regarding the estimates owing to the small number of participants completing this section of the diary.
Time off work because of illness has two separate, different effects: first, on personal costs; and second, on lost productivity. There is an impact on personal costs if participants' take-home pay is directly affected, for example if they are self-employed or are agency workers. There is also a societal cost associated with time off work, which is an estimate of lost productivity that occurred irrespective of whether there was a direct loss of earnings by the participant. In this study, time off work was greater in the waiting list group than the intervention group (Table 3), resulting in a greater productivity loss. Direct out-of-pocket loss of earnings was also higher for this group.

More patients in the CBT group recovered than in the waiting list group (42\% v. 26\%) and QALY gain was greater (mean 0.530, s.d. $=0.099 v$. mean 0.496 , s.d. $=0.126)$.

\section{Cost-effectiveness analysis}

The cost-effectiveness analysis in Tables 5, 6, 7 and 8 is from the NHS perspective, combining the information on cost and outcomes to form ratios of cost per extra patient recovering and cost per QALY gain. This analysis is based on participants for whom we had complete NHS cost and outcome data $(n=133)$, which accounts for the differences between these estimates and those in Table 4. Most differences are small, although the cost of the intervention is a notable exception because those participants for whom we had complete NHS cost data used more sessions of therapy on average (mean 8.0, s.d. $=3.0$ ) than did the full sample (mean 6.1, s.d. $=3.8$ ).

The incremental cost is the extra cost of treating patients in the intervention group, taking into account the cost of the CBT and the cost of other services used. This cost ( $£ 469,95 \%$ CI

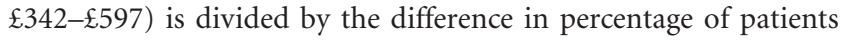
recovering (mean $13.3 \%$, s.d. $=-3.4 \%$ to $30.0 \%$ ) to arrive at an estimate of cost per extra patient recovering ( $£ 3528$ ). Similarly, dividing the difference in cost by the difference in QALYs gives $\mathfrak{E} 17173$ per QALY gain.

Confidence intervals for the estimates of incremental cost and outcome are shown in Table 6. The uncertainty about the estimate of cost per QALY gain is illustrated in Fig. 1, the cost-effectiveness plane that shows the bootstrapped replicates. The degree of spread is an indication of the variability of the point estimates of $£ 469$ and 0.027 QALYs. This is indicated on the chart and the line from the origin through this point represents all other values of $£ 17173$ per QALY gain. All points below this line are less than $£ 17173$ and

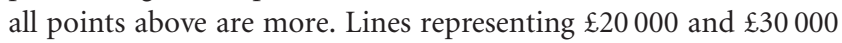
per QALY are shown, as these benchmarks have been identified by NICE as important thresholds in making recommendations about

\begin{tabular}{|c|c|c|c|c|c|c|c|}
\hline & \multicolumn{3}{|c|}{ Waiting list } & \multicolumn{3}{|c|}{ Intervention } & \multirow[b]{2}{*}{ Difference $(95 \% \mathrm{Cl})$} \\
\hline & Cases, $N$ & $n(\%)$ & Mean (s.d.) & Cases, $N$ & $n(\%)$ & Mean (s.d.) & \\
\hline \multicolumn{8}{|l|}{ Cost } \\
\hline Cognitive-behavioural therapy, $£$ & & & & 149 & & $382(238)$ & \\
\hline NHS service use, $f$ & 64 & & $294(355)$ & 73 & & $269(308)$ & $-25(-87$ to 137$)$ \\
\hline Personal expenditure, $€$ & 22 & & $108(147)$ & 30 & & $210(672)$ & $102(-191$ to 396$)$ \\
\hline Out-of-pocket loss of earnings, ${ }^{a} f$ & 64 & & $390(1330)$ & 73 & & $164(964)$ & $-227(-163$ to 616$)$ \\
\hline 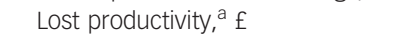 & 66 & & 845 (1872) & 74 & & 514 (1309) & $-330(-866$ to 205$)$ \\
\hline \multicolumn{8}{|l|}{ Consequence } \\
\hline 'Recovered' (BDI <10) & 101 & $26(26)$ & & 109 & $46(42)$ & & 16.5 (3.7 to 29.2 ) \\
\hline BDI score & 101 & & $22.2(15.2)$ & 109 & & $14.7(11.6)$ & $-6.2(-9.3$ to -3.9$)$ \\
\hline QALYS & 77 & & $0.496(0.126)$ & 88 & & $0.530(0.099)$ & $0.034(-0.001$ to 0.069$)$ \\
\hline
\end{tabular}

BDI, Beck Depression Inventory; NHS, National Health Service; QALYS, quality-adjusted life-years.

a. Loss of earnings and the cost of time of work (lost productivity) provide different perspectives of the effect of time off work due to illness. It is misleading to total these as this would result in some double-counting. 


\begin{tabular}{|c|c|c|}
\hline & Waiting list $(n=61)$ & Intervention $(n=72)$ \\
\hline All primary care, f: mean (s.d.) & $164(130)$ & $134(122)$ \\
\hline Hospital, f: mean (s.d.) & $82(222)$ & $95(247)$ \\
\hline Medication, f: mean (s.d.) & $49(101)$ & $43(95)$ \\
\hline NHS cost excluding CBT, f: mean (s.d.) & 295 (359) & $271(310)$ \\
\hline Cost of CBT, f: mean (s.d.) & $0(0)$ & $493(185)$ \\
\hline Total cost NHS perspective, f: mean (s.d.) & 295 (359) & $764(380)$ \\
\hline Recovery $(\mathrm{BDI}<10), n(\%)$ & $19(31)$ & $32(44)$ \\
\hline QALYS, mean (s.d.) & $0.495(0.016)$ & $0.522(0.012)$ \\
\hline Incremental cost, $£(95 \% \mathrm{Cl})$ & & 469 (342 to 597 ) \\
\hline Incremental benefit, difference in percentage recovering $(95 \% \mathrm{Cl})$ & & $13.3(-3.4$ to 30$)$ \\
\hline Incremental benefit, QALY gain $(95 \% \mathrm{Cl})$ & & $0.027(-0.012$ to 0.066 \\
\hline ICER, cost per extra patient recovering & & f3528 \\
\hline ICER, cost per QALY gain & & £17173 \\
\hline \multicolumn{3}{|l|}{ Median net monetary benefit (probability that $>0$ ) } \\
\hline Willingness to pay $(\lambda)=£ 20000$ per QALY & & f70 (0.56) \\
\hline Willingness to pay $(\lambda)=£ 30000$ per QALY & & f336 (0.71) \\
\hline
\end{tabular}

the use of a new healthcare intervention. The NICE guidelines

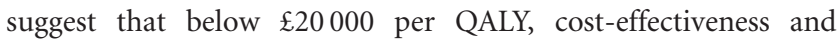
acceptability are the main factors influencing the decision and interventions at this level have a high probability of being

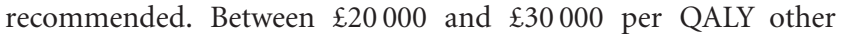
factors, including the degree of uncertainty around the estimate of cost-effectiveness, are considered. Above $£ 30000$ per QALY the intervention is unlikely to be recommended unless there is evidence that there are substantial other benefits not captured by the QALYs. ${ }^{17}$ The cost-effectiveness acceptability curve shown in Fig. 2 can be used to identify the probability of the intervention being cost-effective for any given cost per QALY gain. For example, the point estimate of about $£ 17000$ is represented by a probability of 0.5 because this is the centre of the cost-effectiveness plane (Fig. 1). Figure 2 shows that there is a $56 \%$ chance that online CBT is cost-effective at the $£ 20000$ per QALY

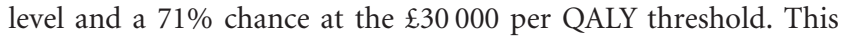
is also represented on Fig. 1 by $56 \%$ and $71 \%$ of the replicates falling below the dotted lines representing these levels of cost per QALY.

\begin{tabular}{|c|c|c|}
\hline & Waiting list $(n=148)$ & Intervention ( $n=149$ ) \\
\hline $\begin{array}{l}\text { NHS costs, f: mean (s.d.) } \\
\text { Incremental cost }(95 \% \mathrm{Cl})\end{array}$ & 300 (268) & $\begin{array}{l}645 \text { (337) } \\
345 \text { (276 to } 415)\end{array}$ \\
\hline $\begin{array}{l}\text { Personal costs, f: mean (s.d.) } \\
\text { Incremental cost }(95 \% \mathrm{Cl})\end{array}$ & 518 (1081) & $\begin{array}{l}410(829) \\
-107(-328 \text { to } 112)\end{array}$ \\
\hline $\begin{array}{l}\text { Value of time off work, } f \text { : mean (s.d.) } \\
\text { Incremental cost }(95 \% \mathrm{Cl})\end{array}$ & 1564 (1790) & $\begin{aligned} & 731(1278) \\
&-834(-1189 \text { to } 479)\end{aligned}$ \\
\hline $\begin{array}{l}\text { QALY gain, f: mean (s.d.) } \\
\text { Incremental benefit }(95 \% \mathrm{Cl})\end{array}$ & $0.494(0.099)$ & $\begin{array}{l}0.528(0.081) \\
0.034(0.014 \text { to } 0.055)\end{array}$ \\
\hline $\begin{array}{l}\text { Incremental cost-effectiveness ratio, cost per QALY gain (NHS perspective) } \\
\text { Median net monetary benefit } P(\mathrm{NMB}>0)\end{array}$ & & f10083 \\
\hline $\begin{array}{l}\text { Willingness to pay }(\lambda)=£ 20000 \text { per QALY } \\
\text { Willingness to pay }(\lambda)=£ 30000 \text { per QALY }\end{array}$ & & $\begin{array}{l}£ 335(0.94) \\
£ 676(0.98)\end{array}$ \\
\hline
\end{tabular}

Table 7 Hospital costs excluding those not related to mental health: sensitivity analysis

\begin{tabular}{|c|c|c|c|c|}
\hline & \multicolumn{2}{|c|}{ Waiting list $(n=64)$} & \multicolumn{2}{|c|}{ Intervention $(n=73)$} \\
\hline & Mean (s.d.) & $\begin{array}{l}\text { Difference (s.d.) from } \\
\text { complete case analysis }\end{array}$ & Mean (s.d.) & $\begin{array}{l}\text { Difference (s.d.) from } \\
\text { complete case analysis }\end{array}$ \\
\hline$A \& E, f$ & $11.4(42.8)$ & $-3.0(17.6)$ & $0(0)$ & $-8.0(25.3)$ \\
\hline Out-patients, $f$ & 53.5 (198.4) & $-13.6(54.2)$ & $53.1(196.0)$ & $-33.5(153.8)$ \\
\hline Total hospital costs, $f$ & $64.9(217.4)$ & $-16.6(56.2)$ & $53.1(196.8)$ & $-41.6(160.2)$ \\
\hline $\begin{array}{l}\text { Incremental cost-effectiveness ratio: cost } \\
\text { per QALY gain (NHS perspective) }\end{array}$ & & & & $£ 16260$ \\
\hline
\end{tabular}




\begin{tabular}{|c|c|c|}
\hline & $\begin{array}{c}\text { Complete case } \\
\text { analysis } \\
(n=133)\end{array}$ & $\begin{array}{l}\text { Imputed data } \\
\qquad(n=297)\end{array}$ \\
\hline Number of CBT sessions, mean & 7.9 & 6.1 \\
\hline Rate used in main analysis, $\mathrm{f}$ & 62.50 & 62.50 \\
\hline Cost per QALY $=£ 20000, £$ & 72 & 118 \\
\hline Cost per QALY $=£ 30000, £$ & 107 & 174 \\
\hline
\end{tabular}

\section{Sensitivity analyses}

Results of the sensitivity analyses are shown in Tables 6,7 and 8 . Table 6 gives the cost and QALY estimates using imputed data. The most notable effect is the reduced estimate of cost to the NHS, which is largely because the overall mean number of sessions used by all participants is less than the number used by the participants for whom we had complete NHS cost data.

The estimate of cost per QALY using imputed data indicates that the base case estimate of $£ 17173$ is conservative. Using the imputed data there is a $94 \%$ chance that the intervention is cost-effective at $£ 20000$ per QALY. Similarly, excluding secondary care that was unlikely to be related to mental health reduces cost per participant in the CBT group by more than that in the waiting list control group (Table 7) suggesting, again, that the base case estimate of $£ 17173$ is conservative. Table 8 shows the effect of varying the cost of the CBT. The results imply that a course of six treatment sessions (the mean for all participants in the trial)

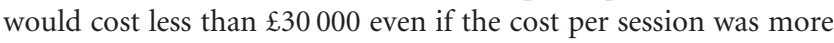
than doubled.

\section{Discussion}

The results of this evaluation suggest that therapist-delivered online CBT is likely to be cost-effective compared with usual care if society is willing to pay at least $£ 20000$ per QALY. This method of delivering CBT has been shown to be effective ${ }^{11}$ and endorsed by Simon \& Ludman, ${ }^{29}$ but their commentary also highlights the importance of cost when using new communication technologies to deliver psychological therapy and here we show that the cost of providing online CBT represents good value for money. In

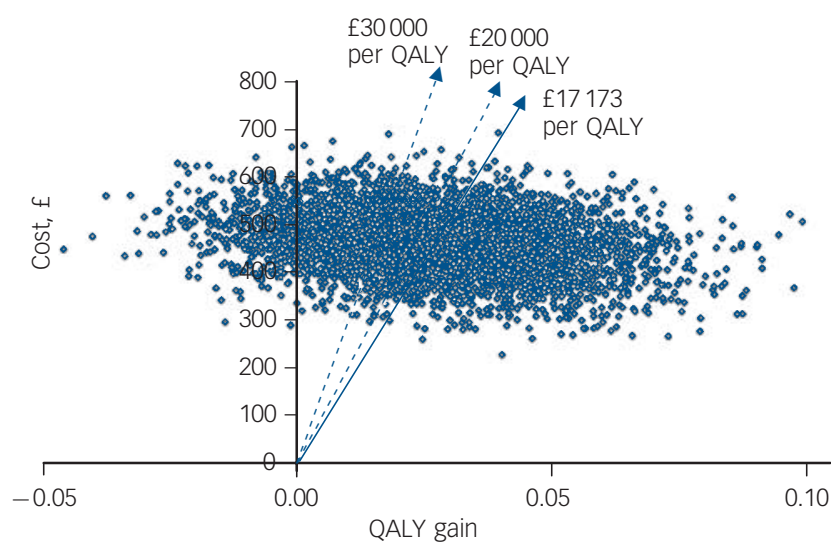

Fig. 1 Cost-effectiveness plane with bootstrapped replicates. QALY, quality-adjusted life-year. addition, we found a societal benefit from the CBT in terms of reduced time off work and consequent productivity gain.

\section{Previous studies}

Although both face-to-face and computerised CBT have been assessed for different patient groups, there have been few analyses of the cost-effectiveness of this therapy: a recent review of the cost-effectiveness of psychological treatments for depression included just three papers on CBT, one of which was more than 15 years old. ${ }^{30}$ The review was unable to draw any firm conclusions about the cost-effectiveness of CBT and observed that most studies had methodological shortcomings. There are, however, two important studies with which to compare our results. First, face-to-face CBT for anxiety and depression was evaluated by Bower et $a l^{31}$ in a cost-effectiveness study alongside a three-arm randomised controlled trial. Face-to-face CBT was effective at 4 months, although this effect was not maintained at 12-month follow-up. No difference in service use or societal costs was detected at either time point. Second, McCrone et al evaluated the cost-effectiveness of computerised CBT for anxiety and depression in primary care patients. ${ }^{9}$ By 8 -month follow-up, computerised CBT patients had used more services than those receiving usual care but, as with our study, lost employment costs were lower. Cost-effectiveness was assessed by comparing cost with BDI scores and the authors concluded that if a one-point improvement is worth $\mathfrak{E} 40$ (2000 prices), this form of CBT is cost-effective. This lends face validity to our result of about $\mathfrak{E} 75$ per point improvement in the BDI given that the computerised CBT was provided at $\mathfrak{E} 14.50$ per session (2000 prices) and our online therapy sessions were costed at $\mathfrak{E} 62.50$ each (2007 prices).

\section{Limitations}

As with many economic evaluations conducted alongside clinical trials, the quality of the resource use data could potentially limit the usefulness of the results. There is debate about the most reliable method of collecting resource use data; ${ }^{32}$ given that there is no consensus about this, the decision is generally guided by pragmatic concerns such as the characteristics of the patient group and resources available. In this study we collected resource use data using a diary, which was judged to be adequate for this patient group. Fewer than $20 \%$ of participants completed all sections of the diary at both time points, although the most complete sections were those asking about NHS resources. Overall, $46 \%$ of participants completed all sections relating to the NHS perspective and an additional $18 \%$ completed the diary at one of the two time points, allowing this information to be used in the imputation. Primary care data, which formed the largest

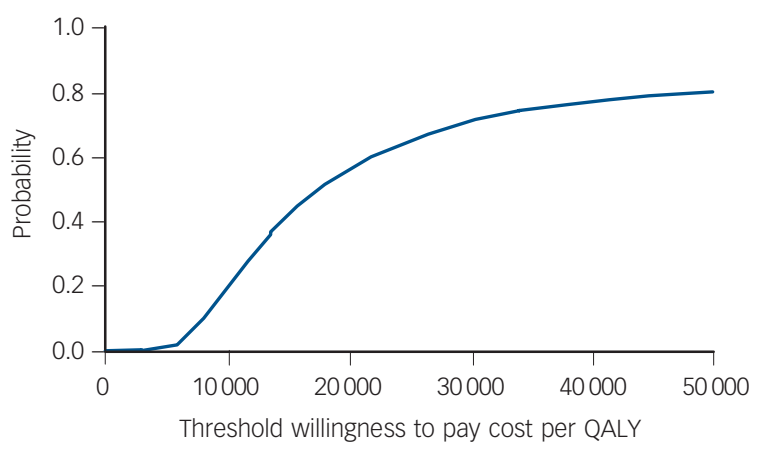

Fig. 2 Cost-effectiveness acceptability curve: probability that the intervention is cost-effective (NHS perspective). QALY, quality-adjusted life-year. 
category of cost to the NHS, were available for at least one of the time periods for $83 \%$ of participants. We acknowledge that more complete data would have been available if we had used questionnaires completed face to face or data from practice records. However, the results of the imputation suggest that any information lost is unlikely to have a major influence on the results or conclusions.

The sections of the diary asking about personal costs were subject to most missing data and here it was often difficult to distinguish between 'missing' and 'none'. It is likely that participants completed the relevant sections of the diary when a cost had been incurred and left them blank (rather than stating 'none') when there had been no expenditure and we enforced a protocol to that effect when interpreting the diary entries. We realise that this category of results is based on data of relatively poor quality, and this is common with personal cost data. Nevertheless, we believe this is not a good reason for ignoring personal costs. Self-management of health is increasingly advocated, particularly in primary care, so it is important to include a patient/family perspective in an economic analysis unless it is clearly not relevant, even if it is difficult and the data are of lower quality.

The difference in the number of CBT sessions received by responders and non-responders is intuitively sensible as it is likely that those participants who used the service most were also more engaged with the trial procedures and completed their diaries more conscientiously. It is possible that participants who dropped out of treatment may have compensated by using other services, but the cost of the intervention is dominant so we believe that our estimates, based on complete data, are likely to overestimate the true cost to the NHS.

\section{Implications for policy}

The results of this study suggest that therapist-delivered online CBT could feasibly be provided by the NHS for primary care patients with depression. The need for better access to CBT has been highlighted by the NHS Improving Access to Psychological Therapy programme ${ }^{8}$ and as an advisor to the programme, Layard has appealed for more training of therapists and suggests they 'should be organised in . . a s similar a way as possible to the clinical trials that showed it to be effective. ${ }^{33}$ This comment refers to therapy clinics but equally could be applied to novel, effective modes of delivery such as evaluated here. Advantages of online CBT compared with a face-to-face service include the reduction in travel time and cost, and the greater flexibility of access, which effectively increase availability. For example, patients who are in employment and reluctant to take time off work often access the service during evenings and weekends. The cost of implementing widespread access to online CBT is unknown. The infrastructure used by NHS Direct could conceivably be used, which would minimise development and set-up costs, and our sensitivity analysis indicates that a realistic rate could be paid for the cost to remain within the threshold of $£ 20000$ per QALY gain.

Providing online CBT in this way is more expensive than computerised CBT, but in a recent review of computerised CBT it was found that a median of only $56 \%$ of trial participants completed the full course and 'significant' staff time was needed to support users. ${ }^{34}$ This suggests that although computerised CBT may be a cost-effective choice for some, there are many patients for whom it is likely to be costly and not very effective. Similarly, online CBT will not be suitable for all. Familiarity with computers is necessary, and qualitative work carried out alongside this trial found that the absence of visual clues may exacerbate the negative thoughts associated with depression in some patients. ${ }^{35}$
On the other hand, the study found that this type of therapy appeals in particular to those who like to write their feelings down, those who value the opportunity to review and reflect on the dialogue of the therapy session, and those who prefer the anonymity offered by this method of delivering CBT. From a provider perspective it may be attractive as an alternative to face-to-face treatment for those whose first language is not English. This could be done by providing access to therapists fluent in other languages based elsewhere in the UK or abroad. It may also appeal when travel is difficult or expensive because of rurality, disability or social phobia.

Sandra Hollinghurst, BA, MA, Tim J. Peters, BSC, MSC, PhD, Surinder Kaur, BSC, Academic Unit of Primary Health Care; Nicola Wiles, BSC, PhD, Glyn Lewis, BA, MSC, MBBS, MRCPsych, MSC, PhD, Academic Unit of Psychiatry; David Kessler, BA MSC, MBBS, MRCPSych, MSC, PhD, Academic Unit of Psychiatry; David Kessler, BA,
MBBS, MRCPsych, MRCGP, MD, Academic Unit of Primary Health Care, Department of Community Based Medicine, University of Bristol, UK

Correspondence: Sandra Hollinghurst, 25 Belgrave Road, Bristol BS8 2AA, UK. Email: s.p.hollinghurst@bristol.ac.uk

First received 18 Sep 2009, final revision 25 May 2010, accepted 24 Jun 2010

\section{References}

1 Kessler RC, Berglund P, Demler O, Jin R, Koretz D, Merikangas KR. The epidemiology of major depressive disorder: results from the National Comorbidity Survey Replication (NCS-R). JAMA 2003; 289: 3095-105.

2 National Collaborating Centre for Mental Health. Depression: Management of Depression in Primary and Secondary Care. National Institute for Clinical Excellence, 2004.

3 McCrone P, Dhanasiri S, Patel A, Knapp M, Lawton-Smith S. Paying the Price. The Cost of Mental Health Care in England to 2006. King's Fund, 2008.

4 Hollinghurst S, Kessler D, Peters TJ, Gunnell DJ. The opportunity cost of antidepressant prescribing. BMJ 2005; 330: 999-1000.

5 Medicines and Healthcare products Regulatory Agency. Selective Serotonin Reuptake Inhibitors (SSRIS): Overview of Regulatory Status and CSM Advice Relating to Major Depressive Disorder (MDD) in Children and Adolescents Including a Summary of Available Safety and Efficacy Data. MHRA, 2005 (http://www.mhra.gov.uk/safetyinformation/Safetywarningsalertsandrecalls/ Safetywarningsandmessagesformedicines/CON019494).

6 King M, Sibbald B, Ward E, Bower P, Lloyd M, Gabbay M, et al. Randomised controlled trial of non-directive counselling, cognitive-behaviour therapy and usual general practitioner care in the management of depression as well as mixed anxiety and depression in primary care. Health Technol Assess 2000; 4: $1-83$.

7 Churchill R, Hunot V, Corney R, Knapp M, McGuire H, Tylee A. A systematic review of controlled trials of the effectiveness and cost-effectiveness of brief psychological treatments for depression. Health Technol Assess 2001; 5: $1-173$.

8 Department of Health. Improving Access to Psychological Therapies (IAPT) Commissioning Toolkit. Department of Health, 2010 (http:// www.iapt.nhs.uk/)

9 McCrone P, Knapp M, Proudfoot J, Ryden C, Cavanagh K, Shapiro DA, et al. Cost-effectiveness of computerised cognitive-behavioural therapy for anxiety and depression in primary care: randomised controlled trial. $\mathrm{Br} J$ Psychiatry 2004; 185: 55-62.

10 Gerhards SAH, de Graf LE, Jacobs LE, Severens JL, Huibers MJH, Arntz A, et al. Economic evaluation of online computerised cognitive-behavioural therapy without support for depression in primary care: randomised trial. Br J Psychiatry 2010; 196: 310-8.

11 Kessler D, Lewis G, Kaur S, Wiles N, King M, weich S, et al Therapistdelivered internet psychotherapy for depression in primary care: a randomised controlled trial. Lancet 2009; 374: 628-34.

12 Beck A, Steer R, Brown G. Manual for the Beck Depression Inventory. The Psychological Corporation, 1987.

13 Lewis G, Pelosi AJ, Araya R, Dunn G. Measuring psychiatric disorder in the community: a standardized assessment for use by lay interviewers. Psychol Med 1992; 22: 465-86.

14 Beck J. Cognitive Therapy, Basics and Beyond. Guilford Press, 1995.

15 Blackburn IM, James IA, Milne DL, Baker C, Standart S, Garland A, et al. The revised cognitive therapy scale (CTS-R): psychometric properties. Behav Cogn Psychother 2001; 29: 431-46.

16 Brooks R. Euroqol: the current state of play. Health Policy 1996; 37: 53-72. 
17 National Institute for Health and Clinical Excellence. Guide to the Methods of Technology Appraisal. NICE, 2008 (http://www.nice.org.uk/media/B52/A7/ TAMethodsGuideupdatedJune2008.pdf).

18 Dolan P, Gudex C, Kind P, Williams A. A Social Tariff for EuroQol. Results from a UK General Population Survey. Centre for Health Economics, University of York, 1995.

19 Curtis L. Unit Costs of Health and Social Care 2007. PSSRU, University of Kent, 2008.

20 Salisbury C, Chalder M, Manku-Scott T, Nicholas R, Deave T, Noble S, et al. The National Evaluation of NHS Walk-in Centres. Final Report. University of Bristol, 2002 (http://www.epi.bris.ac.uk/wic/pdf/ WIC\%20Evaluation\%20Report\%20-\%20Final.pdf).

21 Munro J, Nicholl J, O'Cathain A, Knowles E, Morgan A. Evaluation of NHS Direct First Wave Sites: Final Report of the Phase 1 Research. Medical Care Research Unit, University of Sheffield, 2001 (http://www.sheffield.ac.uk/ content/1/c6/02/40/50/nhsd3.pdf)

22 Department of Health. National Schedule of Reference Costs. Department of Health, 2007 (http://www.dh.gov.uk/en/Publicationsandstatistics/ Publications/PublicationsPolicyAndGuidance/DH_4127649).

23 British Medical Association, Royal Pharmaceutical Society of Great Britain. British National Formulary (issue 54). BMJ Group and Pharm Press, 2007 (http://www.bnf.org/bnf/).

24 The Automobile Association. Running cost Tables. AA, 2007 (http:// www.theaa.com/motoring_advice/running_costs/index.html).

25 Office for National Statistics. Annual Survey of Hours and Earnings (ASHE) 2007 Results. ONS, 2007 (http://www.statistics.gov.uk/pdfdir/ashe1107.pdf).

26 Pritchard C, Sculpher M. Productivity Costs: Principles and Practice in Economic Evaluation. Office of Health Economics, 2000.
27 van Buuren S, Boshuizen HC, Knook DL. Multiple imputation of missing blood pressure covariates in survival analysis. Stat Med 1999; 18: 681-94.

28 Briggs AH. A Bayesian approach to stochastic cost-effectiveness analysis. An illustration and application to blood pressure control in type 2 diabetes. Intl J Tech Assess 2001; 17: 69-82.

29 Simon G, Ludman J. It's time for disruptive innovation in psychotherapy. Lancet 2009; 374: 594-5.

30 Bosmans J, van Schaik $D$, de Bruijne $M$, Van Hout $H$, van Marwijk $H$, van Tulder $\mathrm{M}$, et al. Are psychological treatments for depression in primary care cost-effective? J Ment Health Policy Econ 2008; 11: 3-15.

31 Bower $\mathrm{P}$, Byford S, Sibbald B, Ward E, King, M, Lloyd M. Randomised controlled trial of non-directive counselling, cognitive-behavioural therapy, and usual general practitioner care for patients with depression. II: costeffectiveness. BMJ 2000; 321: 1389-92.

32 Glick H, Polsky D, Schulman K. Trial-based economic evaluations: an overview of design and analysis. In Economic Evaluation in Health Care: Merging Theory with Practice (eds M Drummond, A McGuire): 113-40. Oxford University Press, 2001

33 Layard, R. The case for psychological treatment centres. BMJ 2006; 332 1030-2.

34 Waller R, Gilbody S, Waller R. Barriers to the uptake of computerized cognitive behavioural therapy: a systematic review of the quantitative and qualitative evidence. Psychol Med 2009; 39: 705-12.

35 Beattie A, Shaw A, Kaur S, Kessler RC. Primary care patients' expectations and experiences of online cognitive behavioural therapy for depression: a qualitative study. Health Expect 2009; 12: 45-59.

\section{And the winner is ... the loser}

\section{Peter Byrne}

First of all, I want to thank the Academy (of Motion Picture Arts and Sciences) ${ }^{\circledR}$. If you want to win an acting Oscar ${ }^{\odot}$, play someone with psychiatric illness, intellectual disability or addiction. Think of the drama and the certainty that your character will play out a nervous breakdown (whatever that is) in full Technicolor ${ }^{\odot}$. Split personality (cinema's warped version of schizophrenia) won the Oscar ${ }^{\odot}$ for Frederick March in 1931, Ronald Coleman in 1947, and again for Joanne Woodward in 1957. Addiction brought acting honours to Bette Davis (1935), Ray Milland (1945), ${ }^{*}$ Patricia Neal (1963), Elizabeth Taylor (1966), Nicholas Cage (1995), James Coburn (1998), Marion Cotillard (2007), Jeff Bridges (2009) and Mo'Nique (2009). Despite the relative rarity of films that feature a central character with intellectual disability or autism, when your agent calls with this prized (sic) role, just say yes. Ernest Borgnine (1955), * Cliff Robertson (1968), John Mills (1970), Dustin Hoffman (1988)* and Tom Hanks (1994)* romped home. By today's standards, many of these films are clunky: the eponymous Charly (1968) wonders 'why people who would not dream of laughing at a blind or a crippled man would laugh at a moron'.

Being traumatised into madness won the day for Ingrid Bergman (1944), Alec Guinness (1957)* and Christopher Walken (1978).* Depending on your perspective, either faking madness or playing the socialised psychopath delivered the laurels to Jack Nicholson (1975)* and Angelina Jolie (1999). Psychosis has rewarded Peter Finch (1976), Kathy Bates (1990), Anthony Hopkins (1991), ${ }^{*}$ Geoffrey Rush (1996) and Heath Ledger (2008). Unusually, the first and last recipients won their Oscars ${ }^{\circledR}$ posthumously. Obsessive-compulsive disorder gave Jack Nicholson another statuette in 1997; Tim Robbins won in 2003 for a character with indeterminate symptoms, probably schizophrenia. Playing a character with depression might be tough, but the Oscar ${ }^{\odot}$ cheered up Jane Fonda (1971), Timothy Hutton (1980)* and Nicole Kidman (2002). Two recent outstanding performances, by Tom Wilkinson (2007) and Michael Shannon (2008), depicted mania and depression. Though nominated, neither won, beaten by portrayals of psychopathy and psychosis respectively.

The total number of non-honorary acting Oscars ${ }^{\odot}$ awarded to 2009 is 317 : the 32 listed above account for $10 \%$ of winners. Mental health themes do not feature in $10 \%$ of all films, and nine of these winning films ${ }^{*}$ ) also received the Best Picture Oscar ${ }^{\odot}$. The serious point to be made here is that these representations evoke pity for the unfortunate 'victim', save those psychotic few 'empowered' to torture or kill. The key to removing stigma will be believable characters with whom audiences can identify: parity not pity. 